\title{
SYMMETRIES OF HOMOTOPY COMPLEX PROJECTIVE THREE SPACES
}

\author{
MARK HUGHES
}

\begin{abstract}
We study symmetry properties of six-dimensional, smooth, closed manifolds which are homotopy equivalent to $\mathbf{C} P^{3}$. There are infinitely differentiably distinct such manifolds. It is known that if $m$ is an odd prime, infinitely many homotopy $\mathbf{C} P^{3}$ 's admit $\mathbf{Z}_{m}$-actions whereas only the standard $\mathbf{C} P^{3}$ admits an action of the group $\mathbf{Z}_{m} \times \mathbf{Z}_{m} \times \mathbf{Z}_{m}$. We study the intermediate case of $\mathbf{Z}_{m} \times \mathbf{Z}_{m}$-actions and show that infinitely many homotopy $\mathbf{C} P^{3}$ 's do admit $\mathbf{Z}_{m} \times \mathbf{Z}_{m}$-actions for a fixed prime $m$. The major tool involved is equivariant surgery theory. Using a transversality argument, we construct normal maps for which the relevant surgery obstructions vanish allowing the construction of $\mathbf{Z}_{m} \times \mathbf{Z}_{m}$-actions on homotopy $\mathbf{C} P^{3}$ 's which are $\mathbf{Z}_{m} \times \mathbf{Z}_{m}$-homotopy equivalent to a specially chosen linear action on $\mathbf{C} P^{3}$. A key idea is to exploit an extra bit of symmetry which is built into our set-up in a way that forces the signature obstruction to vanish. By varying the parameters of our construction and calculating Pontryagin classes, we may construct actions on infinitely many differentiably distinct homotopy $\mathbf{C} P^{3}$ 's as claimed.
\end{abstract}

\section{INTRODUCTION}

It is well known that there is a one-to-one correspondence between the integers and the set of diffeomorphism classes of six-dimensional, smooth, closed manifolds which are homotopy equivalent to $\mathbf{C} P^{3}$. (See [MY].) Such manifolds shall hereafter be called homotopy $\mathbf{C} P^{3}$ 's. For every integer $k$, there is a unique homotopy $\mathbf{C} P^{3}$, denoted $X_{k}$, with first Pontryagin class $P_{1}\left(X_{k}\right)=(4+24 k) x^{2}$, where $x \in H^{2}\left(X_{k}\right)$ is a generator. Then, $X_{0}$ is the standard $\mathbf{C} P^{3}$. In what follows, all actions shall be effective and smooth.

Some information is known about smooth finite group actions on homotopy $\mathbf{C P} P^{3}$ 's. For instance, in [H2], it is shown that if $\mathbf{D}_{2 m}$ is the dihedral group of order $2 m$, where $m$ is an odd prime such that the projective class group $\widetilde{K}_{0}\left(\mathbf{Z}\left[\mathbf{D}_{2 m}\right]\right)$ has 2 -rank $=0$, then there are infinitely many integers $k$ for which $X_{k}$ admits a $\mathbf{D}_{2 m}$-action. It is also known that infinitely many homotopy $\mathbf{C} P^{3}$ 's admit a $\mathbf{Z}_{m}$-action for almost every prime number $m$. (See [DM].) On the other hand, in [M1], it is shown that if $X_{k}$ admits a smooth, effective $\mathbf{Z}_{m} \times \mathbf{Z}_{m} \times \mathbf{Z}_{m^{-}}$action, for any odd prime $m$, then $k=0$, i.e., $X_{k}=\mathbf{C} P^{3}$. (There is a version of this result for $m=2$ due to Masuda. Indeed, Corollary 5.2 of [M1] states

Received by the editors February 7, 1991.

1980 Mathematics Subject Classification (1985 Revision). Primary 57R65, 57S17, 57S25.

Key words and phrases. Homotopy complex projective space, smooth group action, equivariant surgery theory, equivariant transversality, surgery obstruction, $G$-signature, Pontryagin class. 
that if $\mathbf{Z}_{2} \times \mathbf{Z}_{2} \times \mathbf{Z}_{2}$ acts smoothly on a homotopy $\mathbf{C} P^{3}, X_{k}$, such that the restricted action of any order two subgroup has fixed point set consisting of precisely two components, then $X_{k}=X_{0}=\mathbf{C} P^{3}$. More information about involutions on homotopy $\mathrm{CP}^{3}$ 's can be found in [DMS], where, in particular, it is shown that every $X_{k}$ admits a smooth conjugation type involution, i.e., an involution with fixed point set consisting of a $\mathbf{Z}_{2}$-cohomology $\mathbf{R} P^{3}$.)

In this paper, we shall consider the intermediate case of $\mathbf{Z}_{m} \times \mathbf{Z}_{m}$-actions. We shall show that infinitely many homotopy $\mathbf{C} P^{3}$ 's admit a $\mathbf{Z}_{m} \times \mathbf{Z}_{m}$-action. We also show how a result of Dovermann implies that for nonstandard homotopy $\mathrm{CP}^{3}$ 's, the only possible fixed point set consists of four points. Our main result is:

Theorem A. Let $m$ be a prime number. There are infinitely many integers $k$ for which $X_{k}$ admits a $\mathbf{Z}_{m} \times \mathbf{Z}_{m}$-action. More precisely, given relatively prime integers $p$ and $q$, each of which is $\equiv \pm 1 \bmod m, X_{k}$ admits $a \mathbf{Z}_{m} \times \mathbf{Z}_{m}$-action, where $k=\left(p^{2}-1\right)\left(q^{2}-1\right) / 3$.

The main tool used to prove the above theorems is equivariant surgery theory (see [DP and PR]). The features of this theory which are relevant to our work shall be outlined in the next section. The third section provides the proof of Theorem $\mathrm{A}$ and considerations on the possible fixed point sets.

\section{BACKGROUND}

Let $G$ be a finite group. Equivariant $(G-)$ surgery is a process for constructing $G$-manifolds which are $G$-homotopy equivalent to a given $G$-manifold $Y$. (A homotopy $F: X \times I \rightarrow Y$ is a $G$-homotopy if $F(\cdot, t)$ is a $G$-map for all $t$.) Two major steps are involved in this process.

1. We build a $G$-normal map $(X, f, b)$ with target manifold $Y$. This can be thought of as an approximation to a $G$-homotopy equivalence.

2. We must determine whether or not the obstructions to performing $G$ surgery to a $G$-homotopy equivalence vanish. The process of $G$-surgery converts $X$ to a $G$-manifold $X^{\prime}$ and $f$ to a $G$-map $f^{\prime}: X^{\prime} \rightarrow Y$ which is a $G$-homotopy equivalence.

Before we elaborate on this, we need some definitions.

Definition 2.1. A $G$-manifold is said to satisfy the gap hypothesis if given a nontrivial subgroup $H \subseteq G$ and a component $F$ of $X^{H}$, we have $2 \operatorname{dim} F<$ $\operatorname{dim} X$.

Recall that a smooth $G$-vector bundle is a triple $(E, p, B)$, where $p: E \rightarrow B$ is an ordinary smooth vector bundle such that $E$ and $B$ support smooth $G$ actions and the projection $p$ is a $G$-map. We also require that, given $g \in G$ and $b \in B$, the map restricted to fiber $g: E_{b} \rightarrow E_{g(b)}$ is linear.

At this point, for simplicity, instead of defining $G$-normal maps, we choose to define a special type of $G$-normal map, namely an adjusted $G$-normal map. (See [D1]. The notion of a $G$-normal map can be found, for example, in [H1 or PR].)

Definition 2.2. An adjusted $G$-normal map with target $Y$ is a triple $(X, f, b)$, where 
(1) $X$ is a smooth, oriented, closed $G$-manifold which satisfies the gap hypothesis and is of dimension $\geq 5$. $Y$ is a smooth, oriented, closed $G$-manifold which is simply connected and of the same dimension as $X$.

(2) $f: X \rightarrow Y$ is a smooth, degree $1 G$-map which induces a $G$-homotopy equivalence between the singular sets $X^{s}$ and $Y^{s}$. (Recall that $X^{s}=\{x \in$ $\left.X: G_{x} \neq 1\right\}$.)

(3) $b$ is a stable $G$-vector bundle isomorphism between $T X \oplus f^{*}\left(\eta_{-}\right)$and $f^{*}\left(T Y \oplus \eta_{+}\right)$, for some pair of $G$-vector bundles $\eta_{ \pm}$. That is, there exists a $G$-representation $V$ such that $b$ is a $G$-vector bundle isomorphism between $T X \oplus f^{*}\left(\eta_{-}\right) \oplus(X \times V)$ and $f^{*}\left(T Y \oplus \eta_{+}\right) \oplus(X \times V)$.

We have a further important definition.

Definition 2.3. Let $\eta_{+}$and $\eta_{-}$be $G$-vector bundles over a $G$-manifold $Y$. Assume that given $H \subseteq G$ and $y \in Y^{H}$, we have $\operatorname{dim}\left(\left.\eta_{+}\right|_{y}\right)^{H}=\operatorname{dim}\left(\left.\eta_{-}\right|_{y}\right)^{H}$. Then $\omega: \eta_{+} \rightarrow \eta_{-}$is a $G$-fiber homotopy equivalence if it is a proper, fiber preserving $G$-map such that, given $H \subseteq G$ and $y \in Y^{H}$, the map $\left(\left.\omega\right|_{y}\right)^{H}:\left(\left.\eta_{+}\right|_{y}\right)^{H}$ $\rightarrow\left(\eta-\left.\right|_{y}\right)^{H}$ has degree 1 when extended to one point compactifications.

Using ideas found in $\S 11$ of Chapter 3 in [PR], an adjusted $G$-normal map can be constructed from a $G$-fiber homotopy equivalence over $Y$ provided that certain conditions are met. This shall be carried out in $\S 3$ of this paper.

We mention that the notion of a $G$-fiber homotopy equivalence is usually defined differently. Indeed, given two $G$-bundles $(E, p, X)$ and $\left(E^{\prime}, p^{\prime}, X^{\prime}\right)$ and a $G$-map $f: X \rightarrow X^{\prime}$, a $G$-fiber homotopy over $f$ is a $G$-map $F: I \times E \rightarrow$ $E^{\prime}$ such that $F(t, \cdot)$ is a $G$-map over $f$ for all $t \in I=[0,1]$. ( $I$ is given the trivial $G$-action.) The maps $f_{0}=F(0, \cdot)$ and $f_{1}=F(1, \cdot)$ are then said to be $G$-fiber homotopic over $f$. A $G$-map $u: E \rightarrow E^{\prime}$ over $\operatorname{Id}_{X}$ is a $G$-fiber homotopy equivalence if there is a $G$-map $v: E^{\prime} \rightarrow E$ such that $v u$ and $u v$ are $G$-fiber homotopic to $\operatorname{Id}_{E}$ and $\operatorname{Id}_{E^{\prime}}$ respectively. It is a fact [PR, $\S \S 1-13$ ] that if $\omega$ is as in Definition 2.3, then it induces a $G$-fiber homotopy equivalence in the usual sense $\Omega: S\left(\eta_{+} \oplus(Y \times \mathbf{R})\right) \rightarrow S\left(\eta_{-} \oplus(Y \times \mathbf{R})\right)$, where $S(\cdot)$ denotes the sphere bundle.

Once our adjusted $G$-normal map is constructed, we shall proceed to step 2, which is to determine whether surgery to a $G$-homotopy equivalence is possible.

We first mention that an equivariant map $f: X \rightarrow Y$ is a $G$-homotopy equivalence if and only if $f^{H}: X^{H} \rightarrow Y^{H}$ is an ordinary homotopy equivalence for all $H \subseteq G$. (See [Br1].) Therefore, given our adjusted $G$-normal map $(X, f, b)$, we must convert $X$ to a $G$-manifold $X_{\eta}$ and $f$ to a $G$-map $F: X_{\eta} \rightarrow Y$ such that $F^{H}$ is a homotopy equivalence for all $H \subseteq G$. There is an obstruction to obtaining such a $G$-homotopy equivalence via $G$-surgery.

Proposition 2.4. Let $(X, f, b)$ be an adjusted G-normal map with target $Y$. There is an obstruction $\sigma_{1}(f, b)$ such that if $\sigma_{1}(f, b)=0$, then $(X, f, b)$ is $G$ normally cobordant to an adjusted $G$-normal map $\left(X_{\eta}, F, B\right)$, where $F: X_{\eta} \rightarrow$ $Y$ is a G-homotopy equivalence.

That is, if $\sigma_{1}(f, b)$ vanishes, then $G$-surgery can be used to convert $X$ to $X_{\eta}$ and $f$ to a $G$-homotopy equivalence $F: X_{\eta} \rightarrow Y$. The proof of this proposition may be found in [D1]. (See Corollary 1.1 on p. 853.) Related results involving $G$-normal maps are well known and can be found in [PR and 
R2]. Also, see [BQ]. We note that this surgery is done relative to the singular set $X^{s}$. The obstruction $\sigma_{1}(f, b)$ is an element of the Wall group $L_{n}^{h}(\mathbf{Z}[G], w)$, where $n=\operatorname{dim} Y$ and $w: G \rightarrow \mathbf{Z}_{2}$ is the orientation homomorphism of the $G$-action on $Y$. (See [W]).

It is often easier to deal with $L_{n}^{s}(\mathbf{Z}[G], w)$, the surgery obstruction group for simple homotopy equivalences, instead of $L_{n}^{h}(\mathbf{Z}[G], w)$. These two groups are related by the Rothenberg exact sequence [Sh]:

$$
\cdots \rightarrow L_{n}^{s}(\mathbf{Z}[G], w) \rightarrow L_{n}^{h}(\mathbf{Z}[G], w) \stackrel{\alpha_{G}}{\longrightarrow} H^{n}\left(\mathbf{Z}_{2} ; \mathrm{Wh}(G)\right) \rightarrow \cdots,
$$

where $\operatorname{Wh}(G)$ is the Whitehead group of $G$ and $\alpha_{G}$ is the torsion homomorphism to be considered shortly. The Tate cohomology group $H^{n}\left(\mathbf{Z}_{2} ; \mathrm{Wh}(G)\right)$ is defined as:

$$
\left\{\delta \in \mathrm{Wh}(G): \delta=(-1)^{n} \delta^{*}\right\} /\left\{\tau+(-1)^{n} \tau^{*}: \tau \in \mathrm{Wh}(G)\right\},
$$

where $*$ denotes the conjugation involution based on the orientation homomorphism $w$.

Let us suppose that our adjusted $G$-normal map $(X, f, b)$ with target $Y$ has been constructed from a $G$-fiber homotopy equivalence $\omega: \eta_{+} \rightarrow \eta_{-}$over $Y$. In this situation, the work of Dovermann [D1] and Dovermann-Rothenberg [DR1] can be applied to give us information on $\alpha_{G}\left(\sigma_{1}(f, b)\right) \in H^{n}\left(\mathbf{Z}_{2} ; \mathrm{Wh}(G)\right)$. Given a $G$-fiber homotopy equivalence $\omega$, its generalized Whitehead torsion $\tau(\omega)$ can be defined as an element of the generalized Whitehead group $\widetilde{\mathrm{Wh}}(G)=$ $\bigoplus \mathrm{Wh}\left(N_{G}(H) / H\right)$, where there is one summand for each conjugacy class of subgroups of $G$. With our set up, a formula due to Dovermann can be used to evaluate $\alpha_{G}\left(\sigma_{1}(f, b)\right)$ in terms of $\tau(\omega)$ and in [DR1], a formula for $\tau(\omega)$ is given in terms of an element in the Burnside ring of $G$.

Indeed, in our proof of Theorem A, we shall use an adjusted $G$-normal map constructed in such a way that the generalized Whitehead torsion of $f^{s}$ vanishes. In this case, Dovermann's formula for $\alpha_{G}\left(\sigma_{1}(f, b)\right)$ reduces to a particularly simple form, namely, $\alpha_{G}\left(\sigma_{1}(f, b)\right)=[T \tau(\omega)]$, where $T$ is conjugation on $\widetilde{\mathrm{Wh}}(G)$ (i.e., the ordinary conjugation involution on each summand) and [.] denotes the cohomology class as indicated above. (We note that in [D1], the right-hand side of the equation actually appears as $[T \tau(\varphi)]$, where $\varphi$ is a $G$-fiber homotopy equivalence closely related to our $\omega$. Indeed, there exists a complex $G$-vector bundle $F$ such that $\varphi$ is obtained by adding id: $F \rightarrow F$ to $\omega: \eta_{+} \rightarrow \eta_{-}$. However, the addition formula Corollary 8.15 of [DR 1$]$ implies that $\tau(\varphi)=\tau(\omega)$. We further note that the results of [D1 and DR 1] are written in terms of sphere bundles. The Whitney sum corresponds to fiberwise join. This is not a restriction for us. See $\S \S 1-13$ of [PR].)

Lemma 2.5. Let $G$ be a finite abelian group and $Y$ an even dimensional $G$ manifold on which $G$ preserves orientation. Let $(X, f, b)$ be an adjusted $G$ normal map with target $Y$ constructed as above from a G-fiber homotopy equivalence $\widetilde{\omega}$ such that the generalized Whitehead torsion of $f^{s}$ vanishes. Suppose that $\widetilde{\omega}=\omega \oplus \omega: \eta_{+} \oplus \eta_{+} \rightarrow \eta_{-} \oplus \eta_{-}$, where $\omega: \eta_{+} \rightarrow \eta_{-}$is a G-fiber homotopy equivalence over $Y$. Then $\alpha_{G}\left(\sigma_{1}(f, b)\right)=0$.

Proof. As mentioned above, [DR1] provides a formula for the generalized Whitehead torsion of $\widetilde{\omega}, \tau(\widetilde{\omega})$. The addition formula, Corollary 8.15 of that 
paper, implies that with our set-up, $\tau(\widetilde{\omega})$ is twice an element of $\widetilde{\mathrm{Wh}}(G)$. Therefore, $T \tau(\widetilde{\omega})$ is also a "multiple of 2." At this point, we note that from our geometric set-up, the only nonzero coordinate of $\tau(\tilde{\omega})$ lies in $\mathrm{Wh}(G)$. Now, it is known (see [B1] or [Ba]), that if $G$ is finite abelian and preserves orientation (i.e., the orientation homomorphism $w$ is trivial), then the conjugation involution $*$ is trivial on $\mathrm{Wh}(G)$. Then, since $n=\operatorname{dim} Y$ is even, we have that $H^{n}\left(\mathbf{Z}_{2} ; \mathrm{Wh}(G)\right)=\mathrm{Wh}(G) / 2 \mathrm{Wh}(G)$. Since 2 divides $\tau(\tilde{\omega})$, it divides $[T \tau(\tilde{\omega})]$ implying that $\alpha_{G}\left(\sigma_{1}(f, b)\right)=0$ as claimed. Q.E.D.

Our purpose for introducing the Rothenberg sequence is to show that $\sigma_{1}(f, b)$ $\in L_{n}^{h}(\mathbf{Z}[G], w)$ comes from an element $\sigma_{1}^{s}(f, b) \in L_{n}^{s}(\mathbf{Z}[G], w)$, which will be shown to vanish, thereby guaranteeing that $\sigma_{1}(f, b)=0$, and that surgery to a $G$-homotopy equivalence is possible. Clearly, $\sigma_{1}(f, b)$ will come from some $\sigma_{1}^{s}(f, b)$ if $\alpha_{G}\left(\sigma_{1}(f, b)\right)=0$.

\section{Proof of Theorem A}

In this section, we shall give the proof of

Theorem A. Let $m$ be a prime number. There are infinitely many integers $k$ for which $X_{k}$ admits a $\mathbf{Z}_{m} \times \mathbf{Z}_{m}$-action. More precisely, given relatively prime integers $p$ and $q$, each of which is $\equiv \pm 1 \bmod m, X_{k}$ admits $a \mathbf{Z}_{m} \times \mathbf{Z}_{m}$-action, where $k=\left(p^{2}-1\right)\left(q^{2}-1\right) / 3$.

Our proof will depend upon an appropriate choice of a model $Y$ on which to base our surgery constructions. Given $p$ and $q$ as above, we will construct a $\mathbf{Z}_{m} \times \mathbf{Z}_{m}$-fiber homotopy equivalence over $Y$, and from it, an adjusted $\mathbf{Z}_{m} \times \mathbf{Z}_{m^{-}}$ normal map. A key point will be the use of a particular involution on $Y$ to kill the signature obstruction. Then, we will show that our set-up is such that all obstructions to surgery vanish.

Our model $Y$ and $\mathbf{Z}_{m} \times \mathbf{Z}_{m}$-fiber homotopy equivalence will be constructed so as to satisfy an important technical condition called the Transversality Condition (Definition 3.1) which will allow us to build from them an adjusted $\mathbf{Z}_{m} \times \mathbf{Z}_{m}$-normal map.

First, we set up some notation. Let $G$ be finite. Given any irreducible, real $G$-representation $\psi$, we define $m_{\psi}: R O(G) \rightarrow \mathrm{Z}$ by setting $m_{\psi}(V)$ equal to the multiplicity of $\psi$ in the virtual representation $V .(R O(G)$ denotes the real representation ring of $G$.) Let $d_{\psi}$ denote the dimension of the real division algebra of $\mathbf{R}$-linear $G$-endomorphisms of $\psi, \operatorname{Hom}_{\mathbf{R}}^{G}(\psi, \psi)$. Finally, let $1_{\mathbf{R}}$ denote the real one-dimensional trivial $G$-representation.

Definition 3.1 (Transversality Condition). (See [P2].) Let $\omega: \eta_{+} \rightarrow \eta_{-}$be a $G$-fiber homotopy equivalence over the smooth $G$-manifold $Y$. We say that the transversality condition is satisfied if for each $H \in \operatorname{Iso}(Y)=\left\{G_{y}: y \in Y\right\}$ and each component $Y_{g}^{H} \subseteq Y^{H}$ the following holds. Let $y \in Y_{\alpha}^{H}$. For each real $H$-representation $\psi$ with $m_{\psi}\left(\left.\eta_{-}\right|_{y}\right) \neq 0$ we have

$$
\operatorname{dim} Y_{\alpha}^{H}=m_{1_{\mathbf{R}}}\left(\left.T Y\right|_{y}\right) \leq d_{\psi} m_{\psi}\left(T Y+\eta_{+}-\left.\eta_{-}\right|_{y}\right)+d_{\psi}-1 .
$$

If the transversality condition is met, Petrie's Transversality Lemma tells us that there are no obstructions to moving $\omega$ by a proper $G$-homotopy to a smooth $G$-map $h$ which is transverse to $Y$, the zero-section of $\eta_{-}$. We then 
set $X=h^{-1}(Y), f=\left.h\right|_{X}$, and $b$ is constructed using the $G$-vector bundles $\eta_{ \pm}$. More precisely, for $H \subseteq G$, we set $X^{H}=\left(f^{H}\right)^{-1}\left(Y^{H}\right)$. Note that if a path component $X_{\alpha}^{H}$ lies in $\left(f^{H}\right)^{-1}\left(Y_{\beta}^{H}\right)$, for some component $Y_{\beta}^{H} \subseteq Y^{H}$, then $\operatorname{dim} X_{\alpha}^{H}=\operatorname{dim} Y_{\beta}^{H}$. Since $\omega$ is a $G$-fiber homotopy equivalence, we can choose the orientation of $X$ so that the $G$-map $f$ will be of degree 1 .

At this point, provided that a few other conditions are met, the triple $(X, f, b)$ will be an adjusted $G$-normal map. In the proof of Theorem A, we shall consider these conditions in detail and show how an adjusted $\mathbf{Z}_{m} \times \mathbf{Z}_{m}$-normal map can be constructed from a particular $\mathbf{Z}_{m} \times \mathbf{Z}_{m}$-fiber homotopy equivalence satisfying the transversality condition.

A further preliminary to the proof of Theorem A is to provide a list of conditions which guarantee the vanishing of the surgery obstruction associated to an adjusted $G$-normal map $(X, f, b)$. In our case,

$$
\sigma_{1}(f, b) \in L_{6}^{h}\left(\mathbf{Z}\left[\mathbf{Z}_{m} \times \mathbf{Z}_{m}\right], 1\right) .
$$

The first condition is that $\alpha_{G}\left(\sigma_{1}(f, b)\right)=0$. Then, $\sigma_{1}(f, b)$ comes from a unique element $\sigma_{1}^{s}(f, b) \in L_{6}^{s}\left(\mathbf{Z}\left[\mathbf{Z}_{m} \times \mathbf{Z}_{m}\right], 1\right)$. (Uniqueness does not hold in general, but it does in our case by applying the Rothenberg sequence and using the fact that $H^{\text {odd }}\left(\operatorname{Wh}\left(\mathbf{Z}_{m} \times \mathbf{Z}_{m}\right) ; \mathbf{Z}_{2}\right)=0$. Indeed, $\operatorname{Wh}\left(\mathbf{Z}_{m} \times \mathbf{Z}_{m}\right)$ is torsion free, in particular, having no two-torsion. See [La].) But, according to [B2], $\sigma_{1}^{s}(f, b)=0 \Leftrightarrow \operatorname{Sign}\left(\sigma_{1}^{s}(f, b)\right)=0$ and $c\left(\sigma_{1}^{s}(f, b)\right)=0$, where

$$
\text { Sign: } L_{6}^{s}\left(\mathbf{Z}\left[\mathbf{Z}_{m} \times \mathbf{Z}_{m}\right], 1\right) \rightarrow R\left(\mathbf{Z}_{m} \times \mathbf{Z}_{m}\right)
$$

is the multisignature and

$$
c: L_{6}^{s}\left(\mathbf{Z}\left[\mathbf{Z}_{m} \times \mathbf{Z}_{m}\right], 1\right) \rightarrow \mathbf{Z}_{2}
$$

is the Kervaire-Arf invariant of classical surgery theory. (Here $R\left(f \mathbf{Z}_{m} \times \mathbf{Z}_{m}\right)$ denotes the complex representation ring.)

There is an interesting $S^{1}$-map due to Ted Petrie (see [MeP, p. 74]) which will be used in our constructions. Given a pair of relatively prime integers $p$ and $q$, take integers $a$ and $b$ such that $-a p+b q=1$ and let $t^{i}$ denote the onedimensional complex $S^{1}$-representation, where $t \in S^{1}$ acts on $\mathbf{C}$ by $t \cdot z=t^{i} z$ (complex multiplication). Define $f: t^{-2 p}+t^{-2 q}=V_{+} \rightarrow t^{-2}+t^{-2 p q}=V_{-}$ by $f\left(z_{0}, z_{1}\right)=\left(\bar{z}_{0}^{a} z_{1}^{b}, z_{0}^{q}+z_{1}^{p}\right)$. It can be shown that $f$ is a proper $S^{1}$ map such that $\operatorname{deg} f^{+}=1$, where $f^{+}$is the extension of $f$ to one point compactifications.

We are now ready to handle the proof of Theorem A.

Proof of Theorem A. For $m=2$, the result is a special case of Theorem 3 in [H1]. So, we let $m$ be an odd prime and let $G=\mathbf{Z}_{m} \times \mathbf{Z}_{m}$. We choose our model $Y$ to be $\mathbf{C} P^{3}$ with the following $\mathbf{Z}_{m} \times \mathbf{Z}_{m}$-action.

Let $g$ denote the one-dimensional complex $G$-representation which associates the $1 \times 1$ matrix $\left(\xi^{i}\right)$ to the element $\left(\xi^{i}, \xi^{j}\right) \in G$. (Here, $\xi=e^{2 \pi i / m}$ and we let the generators of $G$ correspond to $(\xi, 1)$ and $(1, \xi)$.) Likewise, $h$ shall denote the $G$-representation which associates $\left(\xi^{j}\right)$ to $\left(\xi^{i}, \xi^{j}\right)$. Then, $A=g+h+g^{-1}+h^{-1}$ is a $G$-representation and we shall take our model $Y$ to be $p(A)$, the space of complex lines in $A . P(A)$ can be thought of as the orbit space $S(A \otimes t) / S^{1}$, where $t$ denotes the $S^{1}$-representation $t^{1}$ using the above 
notation and $S(\cdot)$ denotes the unit sphere of the indicated representation with respect to a $G \times S^{1}$-invariant metric.

Let us look more closely at this action on $P(A)$. Notice that $G$ has $m+1$ nontrivial proper subgroups, each of which is, of course, isomorphic to $\mathbf{Z}_{m}$. We have $H_{01}=1 \times \mathbf{Z}_{m}, H_{10}=\mathbf{Z}_{m} \times 1$, and $\left\{H_{1 i}=\left\langle\left(\xi, \xi^{i}\right)\right\rangle\right\}_{i=1}^{m-1}$. Now, if $i \neq 1, m-1$, then it is easy to see, using the fact that $m$ is prime, that $P(A)^{H_{1 i}}=P(A)^{G}=\left\{p_{i}\right\}_{i=0}^{3}$, where $p_{0}=[1: 0: 0: 0], p_{1}=[0: 1: 0: 0]$, et cetera. Let $Y_{01}=\left\{\left[z_{0}: z_{1}: 0: 0\right] \mid z_{0}, z_{1} \in \mathbf{C}\right\}=\mathbf{C} P^{1}$ and let $Y_{i j}, i, j=$ $0, \ldots, 3$ be similarly defined. Then it is not difficult to see that $\operatorname{Iso}(P(A))=$ $\left\{1, H_{10}, H_{01}, H_{11}, H_{1 m-1}, G\right\}$ and that $P(A)^{H_{10}}=p_{0} \amalg p_{2} \amalg Y_{13}, P(A)^{H_{01}}=$ $Y_{02} \amalg p_{1} \amalg p_{3}, P(A)^{H_{11}}=Y_{01} \amalg Y_{23}$, and $P(A)^{H_{1 m-1}}=Y_{03} \amalg Y_{12}$. Clearly, our model $Y=P(A)$ satisfies the gap hypothesis.

We shall now construct a $G$-fiber homotopy equivalence over $P(A)$ using Petrie's $S^{1}$-map described above. Let $p$ and $q$ be a pair of relatively prime integers each of which are $\equiv \pm 1 \bmod m$ and let $f: V_{+} \rightarrow V_{-}$be Petrie's map. By taking twisted products we can form vector bundles associated to the $S^{1}$ principal bundle $S(A \otimes t) \rightarrow P(A)$; namely, $\eta_{+}=S(A \otimes t) \times_{S^{1}} V_{+}$and $\eta_{-}=$ $S(A \otimes t) \times_{S^{1}} V_{-}$. The $G$-action on $S(A \otimes t) \times V_{ \pm}$given by the representation $A$ in the first coordinate and the identity in the second coordinate induces a $G$-vector bundle structure on the $S^{1}$ orbit spaces $\eta_{ \pm}$. Upon passing to orbit spaces, the map id $\times f: S(A \otimes t) \times V_{+} \rightarrow S(A \otimes t) \times V_{-}$descends to a $G$-map $\omega: \eta_{+} \rightarrow \eta_{-}$. Since $p$ and $q$ are prime to $|G|$, it can be shown that $\omega$ is actually a $G$-fiber homotopy equivalence. We provide some of the details of the verification of this. Take $H \in \operatorname{Iso}(P(A))$ and suppose that $\operatorname{res}_{H} A=$ $n_{0} \psi_{0}+\cdots+n_{3} \psi_{3}$, where $n_{i} \in\{0,1,2\}, \sum_{i=0}^{3} n_{i}=4$, $\operatorname{res}_{H}$ denotes restriction to $H$, and the $\psi_{i}$ 's are complex one-dimensional $H$-representations. Then $P(A)^{H}=\coprod_{i} P\left(n_{i} \psi_{i}\right)=\coprod_{i} \mathbf{C} P^{n_{i}-1}$, where $\mathbf{C} P^{-1}$ denotes the empty set. If $x \in P\left(n_{i} \psi_{i}\right)$, then $\left.\eta_{+}\right|_{x}=\psi_{i}^{-2 p}+\psi_{i}^{-2 q}$ and $\left.\eta_{-}\right|_{x}=\psi_{i}^{-2}+\psi_{i}^{-2 p q}$ as $H$ representations. (Indeed, suppose $a \in S(A \otimes t)$ lies over $x$. Then $g \cdot[a, v]=$ $[g \cdot a, v]=\left[\psi_{i}(g) a, v\right]=\left[a, \psi_{i}(g) v\right]$ for $\left.[a, v] \in S(A \otimes t) \times_{S^{1}} V_{ \pm}.\right)$Since $p$ and $q$ are prime to $|G|$, these $H$-representations shall be free off the origin so long as $\psi_{i} \neq 1_{H}$, the trivial complex $H$-representation. So, for $H=G, H_{11}$, or $H_{1 m-1}$, we have $\eta_{ \pm}^{H}=P(A)^{H}$, thought of as part of the zero section. For $H=H_{10}$ or $H_{01}, \eta_{ \pm}^{H}=\left.\eta_{ \pm}\right|_{\mathbf{C} P^{1}} \amalg$ (two points), and, of course, for $H=1$, $\eta_{ \pm}^{H}=\eta_{ \pm}$. In every case, the fact that $\operatorname{deg} f^{+}=1$ implies that $\operatorname{deg}\left(\left.\omega\right|_{x}\right)^{H}=1$ (when extended to one-point compactifications) as desired.

In order to ensure that the equivariant surgery obstructions which arise vanish, we shall put a bit more symmetry into this $G$-fiber homotopy equivalence before we construct a normal map from it. Specifically, we shall equip $\eta_{ \pm}$with $G$-equivariant involutions in such a way that $\omega$ becomes a $G \times \mathbf{Z}_{2}$-fiber homotopy equivalence. Equivariant transversality shall provide a $G \times \mathbf{Z}_{2}$-manifold and from this, we will construct an adjusted $G$-normal map for which the signature obstruction vanishes.

Notice that $P(A)$ admits a $G$-equivariant, orientation reversing involution $\phi$. Indeed, given $z=\left[z_{0}: z_{1}: z_{2}: z_{3}\right] \in P(A)$, let $\phi(z)=\left[-\overline{z_{2}}:-\overline{z_{3}}: \overline{z_{0}}: \overline{z_{1}}\right]$. Define $\phi^{\prime}: S(A \otimes t) \rightarrow S(A \otimes t)$ as $\phi^{\prime}\left(a_{0}, a_{1}, a_{2}, a_{3}\right)=\left(-\overline{a_{2}},-\overline{a_{3}}, \overline{a_{0}}, \overline{a_{1}}\right)$ and note that $\phi^{\prime}$ is a $G$-map covering $\phi$. Also notice that $\phi^{\prime}$ induces a $\mathbf{Z}_{4}$-action on $S(A \otimes t)$. When passing to the $S^{1}$ quotient, this action is noneffective 
and results in the $\mathbf{Z}_{2}$-action generated by $\phi$. Then $\tilde{\phi}_{ \pm}: \eta_{ \pm} \rightarrow \eta_{ \pm}$defined by $\tilde{\phi}_{ \pm}[a, v]=\left[\phi^{\prime}(a), v\right]$ are involutions which cover $\phi$ and make $\eta_{ \pm}$into $G \times \mathbf{Z}_{2}$ vector bundles. It is easy to check that $\omega \circ \tilde{\phi}_{+}=\tilde{\phi}_{-} \circ \omega$ and therefore, we see that $\phi$ lifts to $\eta_{ \pm}$in such a way that $\omega$ becomes a $G \times \mathbf{Z}_{2}$-fiber homotopy equivalence.

Now, let $\widetilde{\omega}=\omega \oplus \omega: \widetilde{\eta}_{+} \rightarrow \widetilde{\eta}_{-}$, where $\widetilde{\eta}_{ \pm}=\eta_{ \pm} \oplus \eta_{ \pm}$. This is the $G \times \mathbf{Z}_{2}$-fiber homotopy equivalence which we shall work with.

Our next step is to show that the Transversality Condition is satisfied and that we can construct a $G \times \mathbf{Z}_{2}$-manifold $X$ and a $G \times \mathbf{Z}_{2}$-map $f: X \rightarrow P(A)$. First of all, notice that since $\mathbf{Z}_{2}$ acts freely on $P(A)$, for al $y \in P(A)$, the isotropy group $\left(G \times \mathbf{Z}_{2}\right)_{y}$ is just $G_{y}$. Now, the Transversality Condition (Definition 3.1) may be easily verified using the following computations given for each of the isotropy subgroups $H \in \operatorname{Iso}(P(A))$.

If $H=G$, we have isolated fixed points and it is easily checked that $\left.\tilde{\eta}_{+}\right|_{p_{i}}$ and $\left.\tilde{\eta}_{-}\right|_{p_{i}}$ are equivalent as $G$-representations for all $i$.

If $H=H_{10}$, then $P\left(\operatorname{res}_{H_{10}} A\right)=P\left(\rho+1+\rho^{-1}+1\right)$, where $\rho$ denotes the one-dimensional complex $\mathbf{Z}_{m}$-representation which sends a generator to multiplication by $e^{2 \pi i / m}$. Therefore, we compute

$$
\begin{gathered}
\left.\left(T P(A)+\tilde{\eta}_{+}\right)\right|_{p_{0}}=\rho^{-1}+\rho^{-2}+\rho^{-1}+4 \rho^{-2},\left.\quad \tilde{\eta}_{-}\right|_{p_{0}}=4 \rho^{-2}, \\
\left.\left(T P(A)+\tilde{\eta}_{+}\right)\right|_{y}=1_{H}+\rho+\rho^{-1}+4 \cdot 1_{H},\left.\quad \tilde{\eta}_{-}\right|_{y}=4 \cdot 1_{H},
\end{gathered}
$$

where $y$ is any point in $Y_{13}$. For the computation of the isotropy representations over $P(A)$, see Proposition 2.3 in [H1]. The representations over the point $p_{2}$ are conjugate to those over $p_{0}$. The computations for $H=H_{01}$ are analogous.

If $H=H_{11}$, then $P\left(\operatorname{res}_{H} A\right)=P\left(2 \rho+2 \rho^{-1}\right)$ and we compute

$$
\left.\left(T P(A)+\tilde{\eta}_{+}\right)\right|_{y}=1_{H}+2 \rho^{-2}+4 \rho^{-2},\left.\quad \tilde{\eta}_{-}\right|_{y}=4 \rho^{-2},
$$

where $y$ is any point in $Y_{01}$, while the representations over $Y_{23}$ are conjugate to those over $Y_{01}$. The computations for $H=H_{1 m-1}$ are analogous.

As the verification for $H=1$ is trivial, we see that the transversality condition holds. We can therefore construct the $G \times \mathbf{Z}_{2}$-manifold $X$, the degree $1 G \times \mathbf{Z}_{2}$-map $f: X \rightarrow Y$, and the $G \times \mathbf{Z}_{2}$-vector bundle isomorphism $b$ as indicated after Definition 3.1.

We now proceed to construct an adjusted $G$-normal map from the triple $(X, f, b)$. There are two technical matters which must be considered.

First of all, we claim that $\operatorname{Iso}\left(\tilde{\eta}_{+}\right) \subseteq \operatorname{Iso}(P(A))$. Indeed, consider the subgroups $H_{1 i}$ of $G$, where $i=2, \ldots, m-2$. Given $y \in P(A)^{H_{1 i}},\left.\tilde{\eta}_{+}\right|_{y}$ contains no trivial $H_{1 i}$-representations, so $\tilde{\eta}_{+}^{H_{1 i}}$ lies in the zero section. Thus, $\tilde{\eta}_{+}^{H_{1 i}}=\tilde{\eta}_{+}^{G}=\left\{p_{0}, p_{1}, p_{2}, p_{3}\right\}$ and our claim holds. This condition implies that Iso $(X)=\operatorname{Iso}(P(A))$ and it can be shown that $X$ satisfies the gap hypothesis since $P(A)$ does. (See $[\mathrm{PR}]$ for details.)

It remains to show that all fixed point set components can be made simply connected and that $f^{s}: X^{s} \rightarrow P(A)^{s}$ can be made into a $G$-homotopy equivalence. First notice that it follows from our construction that for $H=G, H_{11}$, or $H_{1 m-1}, X^{H}$ is $G / H$-diffeomorphic to $P(A)^{H}$. This is a result of the fact that for these $H, 1_{H}$ is not a subrepresentation of $\left.\tilde{\eta}_{ \pm}\right|_{y}$ for any $y \in P(A)^{H}$. 
Next, let us consider the cases of $H=H_{10}$ and $H=H_{01}$. Set $X_{13}=$ $\left(h^{H_{10}}\right)^{-1}\left(Y_{13}\right)$ and $X_{02}=\left(h^{H_{01}}\right)^{-1}\left(Y_{02}\right)$. First of all, it is not hard to see that every component of $X_{13}$ (resp. $X_{02}$ ) contains a point with isotropy group equal to $H_{10}$ (resp. $H_{01}$ ). Indeed, if this were not the case for one of these components, then it would also be a component of $X^{G}$, which consists of four isolated fixed points. However, this cannot happen since each component of $X_{13}$ and $X_{02}$ is two-dimensional by construction. By applying zero-dimensional $G / H_{10} \times \mathbf{Z}_{2}$ (resp. $G / H_{01} \times \mathbf{Z}_{2}$ ) surgery, we can make $X_{13}$ (resp. $X_{02}$ ) into a connected $G / H_{10} \times \mathbf{Z}_{2}$ (resp. $G / H_{01} \times Z_{2}$ ) manifold. (To see why $X_{13}$ and $X_{02}$ admit $G$-equivariant involutions, note that the involution $\phi: P(A) \rightarrow P(A)$ restricts to give involutions on $Y_{13}$ and $Y_{02}$ which lift to $\left.\tilde{\eta}_{ \pm}\right|_{Y_{13}}$ and $\left.\tilde{\eta}_{ \pm}\right|_{Y_{02}}$ respectively (commuting with the $G$-action).) Then, we perform zero- and onedimensional $G \times \mathbf{Z}_{2}$-surgery on $X$ to render it connected and simply connected. (This can be done relative to all fixed point set components.) There are no obstructions to these surgeries. (See $\S 9$ of [DP] and Chapter 3 of [PR].) It is important to note that the involution on $X$ reverses orientation. That this holds can be seen using the $\mathbf{Z}_{2}$-equivariance of $f$.

More care is needed in carrying out one-dimensional $G$-surgeries on $X_{13}$ and $X_{02}$. (Note that we are no longer performing $G \times \mathbf{Z}_{2}$-surgery.) We consider $X_{13}$ in detail, the case of $X_{02}$ being similar. We need to show that the surgery kernel $\operatorname{Ker}\left\{f_{*}: H_{1}\left(X_{13}\right) \rightarrow H_{1}\left(Y_{13}\right)\right\}$ can be killed by subtracting $G$-handles. Notice that since $Y_{13}$ is simply connected, this kernel is simply $H_{1}\left(X_{13}\right)$, a direct sum of $2 n$ copies of $\mathbf{Z}$, where $n$ is the genus of $X_{13}$. Since $\mathbf{Z}_{m}$ acts on $X_{13}$, we have that $n$ is a multiple of $m$. (See, for instance, Theorem 3 of [Y].)

At this point, we appeal to the famous result of Jacob Nielsen that an orientation preserving action of a cyclic group is determined up to equivalence by its collection of isotropy representations [N]. Notice that $X_{13}$ has two fixed points, namely, $x_{i}=f^{-1}\left(p_{i}\right)$, for $i=1$ or 3 . The stable $G$-vector bundle isomorphism $b$ allows us to identify the isotropy representations above these points. Indeed, we have, $\left.T X_{13}\right|_{x_{1}}=\left.T Y_{13}\right|_{p_{1}}=g^{-2}$ and $\left.T X_{13}\right|_{x_{3}}=\left.T Y_{13}\right|_{p_{3}}=g^{2}$. Nielsen's result then tells us that the action of $\mathbf{Z}_{m}$ on $X_{13}$ is equivalent to the standard "rotational" action on a genus $n$ surface. By this, we mean the action obtained by considering this surface as a two-sphere with $n$ handles attached symmetrically about the equator so that $\mathbf{Z}_{m}$ acts by rotation about the axis through the north and south poles. (Recall that $n$ is a multiple of $m$.) These poles are left fixed, of course, and are the two fixed points of the action.

However, for this action, the $\mathbf{Z}\left[\mathbf{Z}_{m}\right]$-module structure of $H_{1}\left(X_{13}\right)$ is easily seen and it is also easy to see how to kill $H_{1}\left(X_{13}\right)$ by removing $\mathbf{Z}_{m}$-handles. It must further be checked that the stable bundle isomorphism $b$ extends to the new $G$-manifold resulting from these surgeries. That is, we need to have the appropriate bundle data to give us a new normal map on which to continue our surgery constructions. Notice that the $\mathbf{Z}_{m}$-handles mentioned above consist of $S^{1} \times D^{1}$ 's (thickened appropriately via the equivariant normal bundle). Upon removal, $D^{2} \times S^{0}$ 's are glued in (again appropriately thickened). The question is whether or not the bundles over the $S^{1} \times S^{0}$ 's extend over the $D^{2} \times S^{0}$ 's. A priori, this need not be the case (consider the Lie framing of the torus). However, in our set-up the bundles extend. This can be seen by noting that $X$ is at this point simply connected. So, the bundle restricted to each $S^{1}$ already 
extends over a disk inside $X$. We can use these extensions to define the desired extension when the $D^{2} \times S^{0}$ 's are attached to $X$ minus the $\mathbf{Z}_{m}$-handles. (Notice that we are not doing ambient surgery as it would introduce points on the surface not fixed by the subgroup $H$. Rather, we use the fact that the bundles extend over disks as a way to extend the bundles over the new disks which we attach.) Therefore, one-dimensional $G$-surgery is possible on $X_{13}$ and $X_{02}$ converting them into one-connected $G$-surfaces.

Now, since $X_{13}$ and $X_{02}$ are simply connected, closed surfaces, they must be spheres which, by construction, are $G / H$-homotopy equivalent to $Y_{13}$ and $Y_{02}$ respectively (where $H=H_{10}$ and $H_{01}$ respectively). Indeed, $\left.f\right|_{X_{13}}: X_{13} \rightarrow Y_{13}$ is a $G / H_{10}$-map of degree 1 between two-spheres and is hence a homotopy equivalence. Further, it is a $G / H_{10}$-homotopy equivalence since $\left(\left.f\right|_{X_{13}}\right)^{G / H_{10}}$ is trivially an ordinary homotopy equivalence, being a map taking two points onto two points. Similar considerations apply to $X_{02}$.

At this point, we have an adjusted $G$-normal map, which we shall denote by $\left(X^{\prime}, f^{\prime}, b^{\prime}\right)$. Note that $X^{\prime}$ need not admit an involution, but it is $G$-cobordant to $X$ which does admit a $G$-equivariant, orientation reversing involution. We are then brought to step 2 of the surgery process; i.e., the consideration of the surgery obstruction $\sigma_{1}\left(f^{\prime}, b^{\prime}\right)$.

According to the criterion for the vanishing of $\sigma_{1}\left(f^{\prime}, b^{\prime}\right)$ set out previously, there are three things to be demonstrated.

First of all, we must show that $\alpha_{G}\left(\sigma_{1}\left(f^{\prime}, b^{\prime}\right)\right)=0$. According to Lemma 2.5 , this torsion invariant will vanish provided that the generalized Whitehead torsion of $\left(f^{\prime}\right)^{s}, \tau\left(\left(f^{\prime}\right)^{s}\right)$, vanishes. Notice that $\left(X^{\prime}\right)^{s}$ and $Y^{s}$ are connected, consisting of a union of six two-spheres. (Think of the complete graph on four vertices (with the edges having pairwise disjoint interiors) with edges corresponding to two-spheres and vertices corresponding to points.) We have seen that $\left(f^{\prime}\right)^{G},\left(f^{\prime}\right)^{H_{11}}$, and $\left(f^{\prime}\right)^{H_{1 m-1}}$ are $G$-diffeomorphisms. There is a MayerVietoris formula for generalized Whitehead torsion found on p. 67 of [DR1]; namely, say that $f_{i}: A_{i} \rightarrow B_{i}, i=1,2$ are $G$-maps and that $f_{1}$ and $f_{2}$ coincide on $A=A_{1} \cap A_{2}$. Then $\tau\left(f_{1} \cup f_{2}\right)=\left(j_{1}\right)_{*} \tau\left(f_{1}\right)+\left(j_{2}\right)_{*} \tau\left(f_{2}\right)-j_{*} \tau\left(f_{1} \cap f_{2}\right)$, where $j_{i}: B_{i} \rightarrow B_{1} \cup B_{2}$ and $j: B_{1} \cap B_{2} \rightarrow B$ are inclusions. Using this, we see that it suffices to show that $\tau\left(\left.f^{\prime}\right|_{X_{02}^{\prime}}: X_{02}^{\prime} \rightarrow Y_{02}\right)=0=\tau\left(\left.f^{\prime}\right|_{X_{13}^{\prime}}: X_{13}^{\prime} \rightarrow Y_{13}\right)$. However, this fact follows from our set-up. Indeed, in both cases we have a $\mathbf{Z}_{m}$-homotopy equivalence between two-spheres. (Note that $G=\mathbf{Z}_{m} \times \mathbf{Z}_{m}$ is not acting effectively, so we only need consider the effect $\mathbf{Z}_{m}$-action.) Therefore, the vanishing of $\alpha_{G}\left(\sigma_{1}\left(f^{\prime}, b^{\prime}\right)\right)$ follows from the following claim.

Claim 3.2. The generalized Whitehead torsion of a $\mathbf{Z}_{m}$-homotopy equivalence $h: S^{2} \rightarrow S^{2}$ vanishes.

Proof. Note that in this proof, we are actually computing the torsion of $h$ in the equivariant Whitehead group $\mathrm{Wh}\left(S^{2}\right)$, rather than its image in the generalized Whitehead group $\widetilde{\mathrm{Wh}}(G)$ which is what appears in Dovermann's formula. (See [I] or [Lü] for the equivariant Whitehead group.) Now, it is well known that any compact Lie group action on $S^{2}$ is smoothly equivalent to a linear action. (This is a classical result due to Brouwer [B], Kerekjarto [K], and Eilenberg [E].) So, by composing $h$ on the left and right by $G$-diffeomorphisms, we may assume that we have $h: S(V) \rightarrow S(W)$, where $V$ and $W$ are real three-dimensional $\mathbf{Z}_{m}$-representations. (Note that this new $h$ has vanishing torsion if and only 
if the original $h$ does. Indeed, $\tau(f \circ h \circ g)=f_{*}(\tau(h))$, where $f$ and $g$ are assumed to be $G$-diffeomorphisms. This uses the composition formula found, for instance, on p. 64 of [Lü] and the fact that the torsion of a $G$-diffeomorphism vanishes. Further note that $f_{*}$, the homomorphism induced on the equivariant Whitehead groups, is an isomorphism.) Furthermore, since $S(V)$ and $S(W)$ are $\mathbf{Z}_{m}$-homotopy equivalent $S^{2}$ 's, we must have that $V=W=1_{\mathbf{R}}+\left(\rho^{a}\right)_{\mathbf{R}}$, where $\rho$ is as in the preceeding discussion and $a$ is an integer prime to $m$. For example, it is not hard to see that $Y_{02}=S\left(1_{\mathbf{R}}+\left(g^{-2}\right)_{\mathbf{R}}\right)$. So, we need to show that the generalized Whitehead torsion of a $\mathbf{Z}_{m}$-homotopy equivalence $h: S(V) \rightarrow S(V)$ vanishes. Now, $\operatorname{deg} h= \pm 1$, i.e., we have $\operatorname{deg} h^{H}=\operatorname{deg} 1_{S(V)}^{H}$ or $\operatorname{deg} h^{H}=\operatorname{deg} a^{H}$, for $H=1$ or $\mathbf{Z}_{m}$, where $1_{S(V)}$ is the identity map and $a$ is the antipodal map. (Either of which is a $G$-diffeomorphism and therefore having vanishing torsion.) Then, according to Proposition 3.1 on p. 288 of [T], which is essentially an equivariant Hopf Theorem for linear $G$-spheres, $h$ is $G$-homotopic to either $1_{S(V)}$ or $a$. However, the generalized torsion of a $G$ homotopy equivalence is a $G$-homotopy invariant (see Theorem 4.8 of [Lü]). This establishes our claim. I thank the referee for pointing out the fact that $\mathbf{Z}_{m}$-homotopy equivalences between spheres of the same representation can be considered as units in the Burnside ring and that in this case the units are $\{ \pm 1\}$, both of which may be represented by $\mathbf{Z}_{m}$-diffeomorphisms. Q.E.D.

It now remains to show the vanishing of the associated obstruction $\sigma_{1}^{s}\left(f^{\prime}, b^{\prime}\right)$ $\in L_{6}^{s}(\mathbf{Z}[G], 1)$. The first of the two required steps is to show that the signature $\operatorname{Sign}\left(\sigma_{1}^{s}\left(f^{\prime}, b^{\prime}\right)\right)=0$. Here, a useful formula comes into play, namely, $\operatorname{Sign}\left(\sigma_{1}^{s}\left(f^{\prime}, b^{\prime}\right)\right)=\operatorname{Sign}\left(G, X^{\prime}\right)-\operatorname{Sign}(G, P(A))$, where $\operatorname{Sign}(G, \cdot)$ denotes the $G$-signature of Atiyah and Singer. (See [AS and P1].) Now, by definition, it is clear that $\operatorname{Sign}(G, P(A))=0$ as $P(A)$ has no middle dimensional cohomology. To show that $\operatorname{Sign}\left(G, X^{\prime}\right)=0$ we need two facts. The first is that if a $G$-manifold $M$ admits a $G$-equivariant, orientation reversing diffeomorphism, then $\operatorname{Sign}(G, M)=0$. Secondly, we need the fact that $\operatorname{Sign}(G, \cdot)$ is a $G$-cobordism invariant. Our $X^{\prime}$ may not be equipped with such a diffeomorphism, but it is $G$-cobordant to $X$ which does admit such a diffeomorphism, namely, the involution that was built into it. Therefore, $\operatorname{Sign}\left(G, X^{\prime}\right)=0$ as desired.

The second and final step in showing that $\sigma_{1}^{s}\left(f^{\prime}, b^{\prime}\right)=0$ is to show that the Kervaire-Arf invariant $c\left(\sigma_{1}^{s}\left(f^{\prime}, b^{\prime}\right)\right)$ vanishes. This invariant depends only on the initial (nonequivariant) fiber homotopy equivalence used in the construction of our normal map. It is known that the Kervaire-Arf invariant of twice a fiber homotopy equivalence vanishes. This follows from the fact that the KervaireArf invariant can be expressed in terms of the Kervaire-Sullivan classes via Sullivan's characteristic variety formula (p. 152 of [BM]). The primitivity of these classes implies that a normal map obtained from twice a fiber homotopy equivalence will have vanishing Kervaire-Arf invariant. (Also of relevance is the formula due to Masuda [M2] and independently to Schultz [S1] which gives the Kervaire-Arf invariant for certain fiber homotopy equivalences closely related to ours.) As we are working with $\widetilde{\omega}=\omega \oplus \omega$, we do have $c\left(\sigma_{1}^{s}\left(f^{\prime}, b^{\prime}\right)\right)=0$. Together with the preceeding paragraph this shows that $\sigma_{1}^{s}\left(f^{\prime}, b^{\prime}\right)=0$.

Therefore, we have that $\sigma_{1}\left(f^{\prime}, b^{\prime}\right)$ vanishes and according to Proposition 2.4, $G$-surgery provides us with an adjusted $G$-normal map $\left(X_{\tilde{\eta}}, F, B\right)$, where $F: X_{\tilde{\eta}} \rightarrow P(A)=\mathbf{C} P^{3}$ is a $G$-homotopy equivalence. 
The stable bundle isomorphism $B$ between $T X_{\tilde{\eta}}$ and $F^{*}\left(T Y+\tilde{\eta}_{+}-\tilde{\eta}_{-}\right)$ allows us to compute the Pontryagin class of the smooth manifold $X_{\tilde{\eta}}$. In particular, the first Pontryagin class is given by

$$
p_{1}\left(X_{\tilde{\eta}}\right)=\left(4+8\left(p^{2}-1\right)\left(q^{2}-1\right)\right) x^{2},
$$

where $x \in H^{2}\left(X_{\tilde{\eta}}\right)$ is a generator. (See [H1, $\S \S 6$ and 7] for more details on this calculation.) So, $X_{\tilde{\eta}}=X_{k}$, where $k=\left(p^{2}-1\right)\left(q^{2}-1\right) / 3$ and by varying $p$ and $q$ (within the constraints that $p$ and $q$ are relatively prime and are each $\equiv \pm 1 \bmod m)$ we can construct $\mathbf{Z}_{m} \times \mathbf{Z}_{m}$-actions for infinitely many $k$. Q.E.D.

Remark. Notice that our $\mathbf{Z}_{m} \times \mathbf{Z}_{m}$-actions on $X_{k}$ are such that the fixed point sets consist of four isolated fixed points. It follows from a theorem due to Dovermann that, for $m$ odds, this is the only fixed point set that can arise.

Theorem (Dovermann [D2]). If $\mathbf{Z}_{m}$ ( $m$ an odd prime) acts on $X_{k}$ with fixed point set $F \amalg$ point, where $F$ is connected, then $k=0$. (Note that $F$ must have dimension 4 by Bredon's Theorem [ $\mathrm{Br} 2$, Chapter 7].)

Actually, his statement is more general than this. We have rephrased his result for our purposes. A similar result for $m=2$ is due to Masuda [M1]. Also see [DMSu].

Corollary 3.3. If $\mathbf{Z}_{m} \times \mathbf{Z}_{m}$ ( $m$ an odd prime) acts effectively on $X_{k}$ with $k \neq 0$, then $\left(X_{k}\right)^{\mathbf{Z}_{m} \times \mathbf{Z}_{m}}$ consists of four isolated fixed points.

Proof. Suppose that $G=\mathbf{Z}_{m} \times \mathbf{Z}_{m}$ acts on $X_{k}$ with $k \neq 0$. First of all, $X_{k}^{G}$ cannot be empty since it must have Euler characteristic $\equiv 4 \bmod m$. (This follows from results in Chapter 3 of [Br2].) Next, note that since $\operatorname{dim} X_{k}$ is even and the order of $G$ is odd, each component of $X_{k}^{G}$ must have even dimension. (Indeed, $\nu\left(X_{k}^{G}, X_{k}\right)$ is a sum of irreducible nontrivial $G$-representations and is hence even dimensional.) Now, according to Chapter VI, $\S 3$ of [Hs] every component of $X_{k}^{G}$ must be a $\bmod m$ cohomology $\mathbf{C} P^{r}$, with $r=0,1$, or 2 . If any $\bmod m$ cohomology $\mathbf{C} P^{2}$ occurs, then Dovermann's result implies that $k=0$. So, we may suppose that this does not happen. Next, suppose that $X_{k}^{G}$ contains a $\bmod m$ cohomology $\mathbf{C} P^{1}$. In [Hs], we learn that there is a linear action of $\mathbf{Z}_{m} \times \mathbf{Z}_{m}$ on $\mathbf{C} P^{3}$ which provides a model, up to $\bmod m$ cohomology type, of the orbit structure of our action on $X_{k}$. Now, any linear $G$-action on $\mathbf{C} P^{3}$ leaving a $\mathbf{C} P^{1}$ fixed would have to look like $P\left(\psi+\psi+\psi_{1}+\psi_{2}\right)$, where $\psi, \psi_{1}$, and $\psi_{2}$ are irreducible complex $G$-representations. Note that $\psi \neq \psi_{i}$ for $i=1,2$, but we may have $\psi_{1}=\psi_{2}$. The irreducible character $\psi \circ \psi_{1}^{-1}: G \rightarrow S^{1}$ must have kernel of rank 1 . Therefore, there is a subgroup $K$, isomorphic to $\mathbf{Z}_{m}$, for which $\operatorname{res}_{K} \psi=\operatorname{res}_{K} \psi_{1}$ as $K$-representations. Then, we must have a group isomorphic to $\mathbf{Z}_{m}$ acting on $X_{k}$ and fixing a $\bmod m$ cohomology $\mathbf{C} P^{2}$ or all of $X_{k}$. This forces $k=0$ which is contrary to assumption. Thus, we have no $\bmod m$ cohomology $\mathbf{C} P^{1}$ fixed point set components and the fixed point set consists of isolated fixed points (being a closed submanifold of $X_{k}$ ). The above-mentioned linear model shows that there are four of them. Q.E.D. 
So, for instance, we could not have constructed $\mathbf{Z}_{m} \times \mathbf{Z}_{m}$-actions on nonstandard homotopy $\mathbf{C} P^{3}$ 's by basing our surgery constructions on a model like $P(2 g+2 h)$, which has fixed point set consisting of two copies of $\mathbf{C} P^{1}$.

\section{REFERENCES}

[AS] M. F. Atiyah and I. M. Singer, The index of elliptic operators. III, Ann. of Math. (2) 87 (1968), 546-604.

[B] L. E. J. Brouwer, Über die Periodischen Transformationen der Krugal, Math. Ann. 80 (1921), 39-41.

[B1] A. Bak, The involution on Whitehead torsion, General Topology Appl. 7 (1977), 201-206.

[B2] _ The computation of surgery obstruction groups of finite groups with 2-hyperelementary subgroups, Lecture Notes in Math., vol. 551, Springer-Verlag, 1976, pp. 384-409.

[Ba] H. Bass, $L_{3}$ of finite abelian groups, Ann. of Math. (2) 99 (1974), 118-153.

[Br1] G. Bredon, Equivariant cohomology theories, Lecture Notes in Math., vol. 34, SpringerVerlag, 1967.

[Br2] - Introduction to compact transformation groups, Academic Press, New York, 1973.

[BM] G. Brumfiel and I. Madsen, Evaluation of the transfer and the universal surgery classes, Invent. Math. 32 (1976), 133-169.

[BQ] W. Browder and F. Quinn, A surgery theory for $G$ manifolds and stratified sets, Manifolds -Tokyo, Univ. of Tokyo Press, 1973, pp. 27-36.

[tD] T. tom Dieck, Transformation groups and representation theory, Lecture Notes in Math., vol. 766, Springer-Verlag, 1979.

[D1] K. H. Dovermann, Almost isovariant normal maps, Amer. J. Math. 111 (1989), 851-904.

[D2] _ Rigid cyclic group actions on cohomology complex projective spaces, Math. Proc. Cambridge Philos. Soc. 101 (1987), 487-507.

[DM] K. H. Dovermann and M. Masuda, Exotic cyclic actions on homotopy complex projective spaces, J. Fac. Sci., Univ. Tokyo, Sect. IA Math. 37 (1990), 335-376.

[DMS] K. H. Dovermann, M. Masuda, and R. Schultz, Conjugation involutions on homotopy complex projective spaces, Japanese J. Math. 12 (1986), 1-35.

[DMSu] K. H. Dovermann, M. Masuda, and D. Y. Suh, Rigid versus non-rigid cyclic actions, Comment. Math. Helv. 64 (1989), 269-287.

[DP] K. H. Dovermann and T. Petrie, G-surgery. II, Mem. Amer. Math. Soc., no. 260, 1982.

[DR1] K. H. Dovermann and M. Rothenberg, The generalized Whitehead torsion of a G-fiber homotopy equivalence, Transformation Groups, Proc. Conf. Osaka Japan 1987, Lecture Notes in Math., vol. 1375, Springer-Verlag, 1989, pp. 60-88.

[DR2] _ Equivariant surgery and classification of finite group actions on manifolds, Mem. Amer. Math. Soc., no. 397, 1988.

[E] S. Eilenberg, Sur les transformations de la surface de sphere, Fund. Math. 22 (1934), 28-41.

[H1] M. Hughes, Finite group actions on homotopy complex projective spaces, Math. Z. 199 (1988), 133-151.

[H2] _ Dihedral group actions on homotopy complex projective three spaces, Pacific J. Math. 150 (1991), 97-110.

[Hs] W. Y. Hsiang, Cohomology theory of topological transformation groups, Ergeb. Math. Grenzgeb. 85, Springer-Verlag, 1975.

[I] S. Illman, Whitehead torsion and group actions, Ann. Acad. Sci. Fen. 588 (1974).

[K] B. Kerekjarto, Über die periodischen transformationens der Kreisscheibe und der Kugelfläche, Math. Ann. 80 (1921), 36-38.

[La] T. Y. Lam, Induction theorems for Grothendieck groups and Whitehead groups of finite groups, Ann. Sci. École Norm. Sup. (4) 1 (1968), 91-148.

[Lü] W. Lück, Transformation groups and algebraic $K$-theory, Lecture Notes in Math., vol. 1408, Springer-Verlag, 1989. 
[M1] M. Masuda, Smooth involutions on homotopy C $P^{3}$, Amer. J. Math. 106 (1984), 14871501.

[M2] _ The Kervaire invariant of some fiber homotopy equivalences, Adv. Studies in Pure Math., vol. 9, Kinokuniya and North-Holland, 1987.

[MeP] A. Meyerhoff and T. Petrie, Quasi-equivalence of G-modules, Topology 15 (1976), 69-75.

[MY] D. Montgomery and C. T. Yang, Differentiable actions on homotopy seven spheres. I, Trans. Amer. Math. Soc. 112 (1966), 480-498.

[N] J. Nielsen, Die Struktur Periodischer Transformationen von Flächen, Danske Vid. Selsk., Mat.-Fys. Medd. 15 (1937), 1-77.

[P1] T. Petrie, The Atiyah-Singer invariant, the Wall groups $L_{n}(\pi, 1)$ and the function te $e^{x}+$ 1/te -1 , Ann. of Math. (2) 92 (1970), 174-187.

[P2] - Pseudo-equivalences of G-manifolds, Proc. Sympos. Pure Math., vol. 32, Amer. Math. Soc., Providence, R.I., 1978, pp. 169-210.

[PR] T. Petrie and J. Randall, Transformation groups on manifolds, Dekker Lecture Series, vol. 48, Dekker, 1984.

[S1] R. Schultz, private communication to Y. D. Tsai.

[Sh] J. Shaneson, Wall's surgery obstruction groups for $G \times \mathbf{Z}$, Ann. of Math. (2) 90 (1969), 296-334.

[T] J. Tornehave, Equivariant maps of spheres with conjugate orthogonal actions, Algebraic Topology Conf.-London, Ontario, 1981, Canad. Math. Soc. Conf. Proc., vol. 2, Amer. Math. Soc., Providence, R.I., 1982, pp. 275-301.

[W] C. T. C. Wall, Surgery on compact manifolds, Academic Press, New York, 1970.

[Y] K. Yokoyama, Classification of periodic maps on compact surfaces: I, Tokyo J. Math. 6 (1983), 75-94.

Department of Mathematics, Florida State University, Tallahassee, Florida 32306

Current address: Department of Mathematics, Frostburg State University, Frostburg, Maryland 21532

E-mail address: e2mthughes@fre.towson.edu 